\title{
Seismic modelling as a tool for optimization of downhole microseismic monitoring array
}

\author{
Andrzej Pasternacki, Eryk Święch, Tomasz Maćkowski \\ AGH University of Science and Technology, Faculty of Geology, Geophysics and Environmental Protection, \\ Department of Fossil Fuels; al. A. Mickiewicza 30, 30-059 Krakow, Poland; e-mail: anpaster@agh.edu.pl, \\ swiech.eryk@gmail.com, mackowsk@agh.edu.pl
}

(C) 2016 Authors. This is an open access publication, which can be used, distributed and reproduced in any medium according to the Creative Commons CC-BY 4.0 License requiring that the original work has been properly cited.

Hydraulic fracturing processes employed to release natural gas accumulations trapped in shale formation causes cracks in fractured media occurred as microseismic events. Those events can be detected with either surface or downhole monitoring technique. One of the advantages of downhole microseismic monitoring technique is the relative high detection moment magnitude threshold, compared to surface and quasi surface arrays (Maxwell 2014). The epicenters of detected microseismic events are located with certain accuracies (Eisner et al. 2010). The uncertainties in location are mainly caused by simplification of a very complex geological structure, geometry of the monitoring network, arrival time pick uncertainty and naturally selected processing method. The correct assessment of macroseismic events locations with their uncertainties is the key to proper interpretation of the results.

In this study, authors present an analysis of optimizing geometry of the downhole microseismic monitoring array minimalizing location error and taking into account level of detectability. To achieve this goal, several different downhole array geometries were tested.

The study is located in Northern Poland where active exploration of shale gas deposits takes place. In the investigated area three wells are located, one vertical (W-1) and two horizontal, which have been drilled in the same azimuths but different direction and slightly different depths (W3H - deeper and W2Hbis - shallower). As there is possibility that these wells will be stimulated in close period of time, the chosen array placed in the monitoring well should be optimal for depths. As Eisner stated in his work, best downhole array should have to consist of $3 \mathrm{C}$ sensors placed below and above of the planed depths of stimulation to reduce uncertainty of the event locations (Eisner et al. 2009). Both treatment wells have relatively high horizontal distance, which results with high distance between receivers and possible events (in ranges between $500 \mathrm{~m}$ to $1700 \mathrm{~m}$ ), which is quite high compared to literature examples (Warpiński \& Natl 1994).

To perform this analysis, GeoTomo $\mathrm{MiVu}^{\mathrm{TM}}$ Microseismic Processing System was used, which includes a Vecon modeling engine. This software has been granted to AGH UST for research and educational purposes. The passive seismic modelling was done with GRTM method (generalized reflection transmission coefficients) (Kennet 1980). This kind of mixed procedure is relatively fast to perform and allows checking many different configurations of downhole array. Based on the 3D seismic survey provided by PGNiG in the investigated area authors have decided to use simple layered velocity model which sufficiently describes the local geological conditions. The synthetic microseismic events were located using TGS (Traveltime Grid Search) algorithm available in $\mathrm{MiVu}$ software.

Based on presented analysis authors were able to choose optimal geometry of downhole 
microseismic array for both prospective intervals which fulfill condition of being good compromise between costs and location accuracy of possible events.

This study was supported by the Grant of The Polish National Center for Research and Development in the Program Blue Gas, project entitled "Appraisal of microseismic monitoring techniques of hydraulic fracturing and development of optimal processing and interpretation methodologies" (Acronym: SHALEGASMICROS).

\section{REFERENCES}

Eisner L., Duncan P.M., Heigl W.M \& Keller W.R., 2009. Uncertainties in passive seismic monitoring. The Leading Edge, 06, 648-655.

Kennett B., 1980. Seismic waves in a stratified half space II. Theoretical seismograms. Geophysical Journal International, 61, 1, 1-10.

Maxwell S., 2014. Microseismic Imaging of Hydraulic Fracturing: Improved Engineering of Unconventional Shale Reservoirs. Society of Exploration Geophysicists.

Warpiński N.R. \& Natl S., 1994. Interpretation of hydraulic fracture mapping experiments. [in:] University of Tulsa Centennial Petroleum Engineering Symposium, 29-31 August, Tulsa, Oklahoma, SPE-27985-MS, Society of Petroleum Engineers, 1-10. 